\title{
Prevalence of and risk factors associated with the presence of Staphylococcus aureus in the chronic wounds of patients treated in primary health care settings in Brazil
}

\author{
Eliane Patricia Lino Pereira-Franchi[ ${ }^{[1],[2]}$, Maria Rachel Nogueira Barreira[1], \\ Natália de Sousa Lima Moreira da Costa ${ }^{[1]}$, Carlos Magno Castelo Branco Fortaleza ${ }^{[2]}$ \\ and Maria de Lourdes Ribeiro de Souza da Cunha ${ }^{[1]}$
}

\begin{abstract}
[1]. Departamento de Microbiologia e Imunologia, Instituto de Biociências de Botucatu, Universidade Estadual Paulista Júlio de Mesquita Filho, Botucatu, SP, Brasil. [2]. Departamento de Doenças Tropicais, Faculdade de Medicina de Botucatu, Universidade Estadual Paulista Júlio de Mesquita Filho, Botucatu, SP, Brasil.
\end{abstract}

\begin{abstract}
Introduction: Wounds can be colonized by methicillin-resistant Staphylococcus aureus (MRSA). Methods: We evaluated the prevalence of $S$. aureus and MRSA in the wounds of patients treated at Basic Health Units in Brazil and identified risk factors associated with their presence. Results: The prevalence rates of $S$. aureus and MRSA were $51.5 \%$ and $8.7 \%$, respectively. There was a correlation between the presence of $S$. aureus in wounds and nostrils $(p<0.01)$. A positive association was detected between $S$. aureus infection and previous benzylpenicillin use $(p=0.02)$. No associations were observed for MRSA. Conclusions: Multidrug-resistant pathogens are present in primary healthcare settings in Brazil.
\end{abstract}

Keywords: S. aureus. Wounds. Primary healthcare.

Wounds are a risk factor for colonization by pathogenic bacteria, including multidrug-resistant microorganisms and methicillin-resistant Staphylococcus aureus (MRSA) ${ }^{1}$. MRSA interferes with wound healing, increases the severity of the lesions, and increases the risk of other types of infection such as pneumonia or bacteremia. Moreover, infected lesions act as reservoirs and sources of infection of other individuals.

Most published studies of MRSA have been performed in the hospital setting; few have investigated MRSA in primary care facilities. The present study was conducted in the basic health units (BHU) of primary care facilities, where almost $80 \%$ of medical consultations take place. These BHU, coupled with secondary and tertiary care facilities, form the Brazilian healthcare system. Thus, it is crucial to investigate the profile of pathogens such as $S$. aureus that infect the wounds of patients managed in this setting. Given the large number of individuals with infected wounds and the increasing dissemination of MRSA in hospitals and communities, this study aimed to establish the prevalence of and to identify related risk factors for methicillinsensitive Staphylococcus aureus (MSSA) and MRSA strains in the wounds of patients attended to at the BHU.

Corresponding author: Dra. Eliane Patricia Lino Pereira Franchi. e-mail: fliane24@yahoo.com.br

Received 30 May 2017

Accepted 24 August 2017
This cross-sectional study included patients attending any of the 17 BHU in the City of Botucatu, São Paulo, Brazil, for wound care during the period 2010-2013. We included all patients with pre-existing and new wounds (colonized or infected) of any etiology, regardless of patient age. Patients who reported having been hospitalized or having undergone surgery or invasive procedures up to one year prior to sample collection, were excluded.

A sterile swab (Copan Diagnostics Inc., USA) was used to collect material from patients' wounds and nasal cavities ${ }^{2,3}$. After collection, samples were immediately forwarded to the bacteriology laboratory at the State University of São Paulo Júlio de Mesquita Filho [Universidade Estadual Paulista Júlio de Mesquita Filho (UNESP)] for S. aureus identification ${ }^{4}$. Drug susceptibility tests were performed as previously described ${ }^{5}$, with disks containing oxacillin $(1 \mu \mathrm{g})$, cefoxitin $(30 \mu \mathrm{g})$, penicillin $(10 \mathrm{U})$, erythromycin $(15 \mu \mathrm{g})$, clindamycin $(2 \mu \mathrm{g}$; D test), gentamicin $(10 \mu \mathrm{g})$, levofloxacin $(5 \mu \mathrm{g})$, sulfamethoxazole/ trimethoprim $(25 \mu \mathrm{g})$, quinupristin/dalfopristin $(15 \mu \mathrm{g})$, linezolid $(30 \mu \mathrm{g})$, tigecycline $(15 \mu \mathrm{g})$, and fusidic acid $(10 \mu \mathrm{g})$. The minimum inhibitory concentrations (MIC) of oxacillin and vancomycin were determined with E-test ${ }^{\circledR}$ strips (BioMérieux, UK). Vancomycin heteroresistance was screened for using the modified macromethod ${ }^{6}$.

Deoxyribonucleic acid (DNA) was extracted using the Illustra Kit (GE Healthcare, USA) according the manufacturer's 
instructions. S. aureus isolates were classified as MRSA based on the results of real-time polymerase chain reaction (PCR) assays for $m e c \mathrm{~A}$ and $p v l$ in a $20-\mu \mathrm{L}$ volume containing $10 \mu \mathrm{L}$ of Fast SYBR ${ }^{\circledR}$ Green Master Mix (Applied Biosystems, Belgium), $0.6 \mu \mathrm{L}$ of each primer $(250 \mathrm{nM}), 4 \mu \mathrm{L}$ of template, and $4.8 \mu \mathrm{L}$ of MilliQ water. The following thermocycling reaction was performed in a StepOnePlus ${ }^{\mathrm{TM}}$ system (Applied Biosystems): 30 -s pre-heating at $60^{\circ} \mathrm{C}$, followed by $20 \mathrm{~s}$ at $95^{\circ} \mathrm{C}, 40$ cycles of denaturation at $95^{\circ} \mathrm{C}$ for $1 \mathrm{~s}$, and annealing at $60^{\circ} \mathrm{C}$ for $20 \mathrm{~s}$. International reference strains, such as ATCC (American Type Culture Colletion) 33591 (mecA positive), ATCC 25923 (mecA negative), and ATCC 49775 ( $p v l$ positive), were included as controls. The primers have been described elsewhere 7 . A previously described multiplex PCR protocol ${ }^{8}$ was used to characterize mec. The following controls were used for staphylococcal cassete chromosome mec (SCCmec) typing, the COL strain for type I, N315 for type IA, PER34 for type II, AN546 for type III, HU25 for type IIIA, MW2 for type IV, and JCSC for type V.

A structured questionnaire was used to obtain socioeconomic and demographic data. All information was obtained from patient records or from the patients themselves. Colonized and infected wounds were distinguished based on clinical signs and symptoms of infection. Wounds were classified as infected if at least two of the following signs were observed: Fever; local erythema, edema, pain, or heat; and increased amount of purulent secretions 9 .

For the univariate analysis, we used EPI-INFO for Windows, v.7 (Centers for Disease Control and Prevention, USA). Nonparametric tests for proportions ( $\chi^{2}$ or Fisher's exact test, as appropriate) were performed for comparisons of categorical variables. Continuous variables were compared using Student's $t$-test or the Mann-Whitney U test. Multivariate analysis was performed using IBM SPSS Statistics, Version 20.0 (Armonk, NY: IBM Corp). The outcomes of interest were the presence of $S$. aureus or MRSA, independent of the sample collection site. Logistic regression models were built via backward stepwise variable selection. The criterion for including and retaining variables in the model was a $p$-value $<0.05$. The results are expressed as odds ratios (OR) with $95 \%$ confidence intervals (CI).

The study included 171 patients (Table 1), and the prevalence rates of S. aureus and MRSA were $51.5 \%$ and $8.7 \%$, respectively (Figure 1). Of the 119 S. aureus isolates, most were resistant to penicillin $[n=101(85 \%)]$, followed by erythromycin $[n=32(27 \%)]$, gentamicin $[n=14(12 \%)]$, clindamycin $[n=13(11 \%)]$, and levofloxacin $[n=7(6 \%)]$. Resistance to fusidic acid, tigecycline, sulfamethoxazole/trimethoprim, quinupristin/dalfopristin, and linezolid was not detected. Similar resistance profiles were observed in MRSA isolates from several different patients (Table 2). The MIC was $0.12-256 \mu \mathrm{g} / \mathrm{mL}$ for oxacillin and $0.38-2.00 \mu \mathrm{g} / \mathrm{mL}$ for vancomycin. The $\mathrm{MIC}_{50}$ was $0.38 \mu \mathrm{g} / \mathrm{mL}$ for oxacillin and $1.5 \mu \mathrm{g} / \mathrm{mL}$ for vancomycin, while the $\mathrm{MIC}_{90}$ was $24 \mu \mathrm{g} / \mathrm{mL}$ for oxacillin and $2 \mu \mathrm{g} / \mathrm{mL}$ for vancomycin. Eighteen isolates from 15 patients were resistant to oxacillin and cefoxitin; all isolates were sensitive to vancomycin. Vancomycin heteroresistance screening revealed no positive samples. Resistance to cefoxitin was similar to that observed for oxacillin.
In the multivariate analysis, we observed negative associations between the presence of $S$. aureus and age (OR, 0.94; 95\% CI, $0.90-0.98 ; p<0.01)$, amoxicillin use (OR, $0.16 ; 95 \% \mathrm{CI}$, $0.04-0.60 ; p<0.01)$, and ciprofloxacin use (OR, $0.28 ; 95 \% \mathrm{CI}$, $0.08-0.98 ; p=0.04)$; a positive association was observed with benzylpenicillin use (OR, 3.81; 95\% CI, 1.23-11.82; $p=0.02)$. No risk factors for MRSA were identified in the multivariate analysis. Nasal swabs were collected from only 74 (43.3\%) patients; $S$. aureus and MRSA were detected in $28.4 \%$ and $5.4 \%$ of these samples, respectively. S. aureus was isolated from the wounds and nasal cavities of $41(55.4 \%)$ and $21(28.3 \%)$ of these patients, respectively. Associations were found between nasal carriage of $S$. aureus and its presence in the wound $(p<0.01)$ and between nasal carriage of MRSA and its presence in the wound $(p<0.01)$. Associations between antimicrobial drug resistance and the presence of the $p v l$ gene in MSSA and MRSA isolates from wounds, were investigated. Univariate analysis demonstrated positive associations between resistance to levofloxacin (OR, 68; 95\% CI, 6.8-479.4; $p<0.01$ ), clindamycin (OR, 15.5; 95\% CI, 3.3-73.3; $p<0.01$ ), or erythromycin (OR, 6.58; 95\% CI, 1.97-21.93; $p<0.01)$ and MRSA.

The results in this study highlight a poorly addressed issue: The presence of multidrug-resistant pathogens in primary care facilities. We observed prevalence rates of $51.5 \%$ for $S$. aureus and $8.7 \%$ for MRSA; resistance to macrolides, lincosamides, aminoglycosides, and quinolones in sensitive and resistant isolates; and a higher frequency of SCCmec type IV MRSA, followed by type II. Studies addressing the prevalence of MRSA in primary care units are sparse ${ }^{10}$. In Brazil, only two such studies have been conducted; both were performed by the same research group in the City of Goiãnia, Goiás. The first was a microbiological and antimicrobial assessment of the microorganisms present in chronic leg ulcers; $S$. aureus was the major species identified (65\%), and $20.5 \%$ of the isolates were resistant to oxacillin and cefoxitin ${ }^{11}$. The second study demonstrated the presence of antimicrobial-resistant $S$. aureus and coagulase-negative staphylococci isolates from venous ulcers of 68 patients during the period 2009-2010 $0^{12}$. In both studies, prevalence was calculated using the number of samples collected, which equaled the number of ulcers, rather than the study population, making it impossible for the reader to calculate the real prevalence of $S$. aureus and MRSA. Moreover, no risk factor analysis was performed.

The prevalence observed in our study was lower than that observed in hospital-based studies, such as the study by Forcade et al. ${ }^{10}$ - performed in 10 primary care clinics in Texas, USA between 2001 and 2010 - in which MRSA was isolated from $73(63 \%)$ of 119 patients with skin and soft tissue infections. Almeida et al. ${ }^{1}$ investigated the prevalence of $S$. aureus and MRSA in patients in a small hospital in Northeastern Brazil; they reported that $20 \%$ and $6.4 \%$ of wounds were colonized by $S$. aureus and MRSA, respectively. The same study also described associations between wound colonization and nasal carriage, recent antibiotic use, and ward admission. Those data agree with our results, which revealed a positive association between nasal carriage of both $S$. aureus and MRSA and their respective presence in the wound. We also observed that in $31 \%$ 
TABLE 1: Univariate and multivariate analysis of risk factors for carriage of Staphylococcus aureus and MRSA in patients attending basic health units in Botucatu, Sao Paulo State, Brazil.

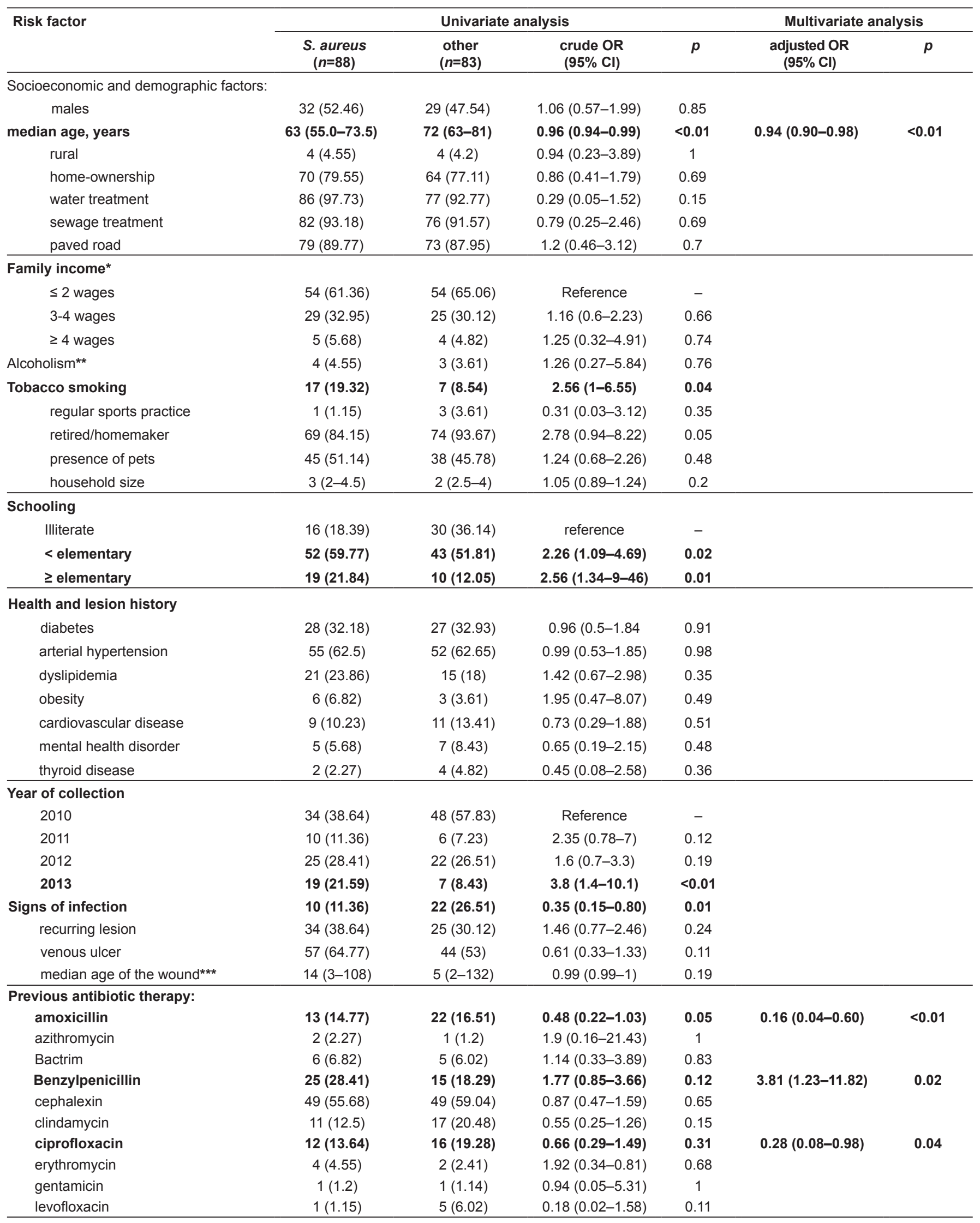

MRSA: methicillin-resistant Staphylococcus aureus; S.: Staphylococcus OR: odds ratio; CI: confidence interval. *Data refer to monthly family income in minimum wages. ${ }^{* *}$ Regular consumption of alcoholic beverages, more than twice a week. ${ }^{* * *}$ Unit of measure: months. All data are expressed as $\mathrm{n}$ (\%), except where specified. Significant results are highlighted in bold. 
TABLE 2: Characterization of MRSA isolates from patients attended to at the BHUs of the City of Botucatu, Sao Paulo State, Brazil.

\begin{tabular}{|c|c|c|c|c|c|}
\hline Patient & MRSA & Type & Resistance & Origin & BHU** \\
\hline 2 & 2 & II & oxa, cfo, Ivx, cli, ery, pen & wound ${ }^{*}$ & $\mathrm{~B}$ \\
\hline 3 & 3 & ॥ & oxa,cfo, Ivx, cli, ery, pen & wound* & $\mathrm{C}$ \\
\hline & 5 & ॥ & oxa, cfo, Ivx, cli, ery, pen & nasal & \\
\hline 5 & 6 & II & oxa, cfo, Ivx, gen, cli, ery, pen & wound* & $E$ \\
\hline 6 & 7 & IV & oxa, cfo, Ivx, cli, ery, pen & wound ${ }^{*}$ & B \\
\hline 8 & 10 & IV & oxa, cfo, pen & wound & $\mathrm{F}$ \\
\hline 9 & 11 & IV & oxa, cfo, pen & wound & G \\
\hline 10 & 12 & IV & oxa, cfo, pen & wound & $\mathrm{H}$ \\
\hline 11 & 13 & IV & oxa, cfo, gen, ery, pen & wound* & C \\
\hline 12 & 14 & IV & oxa, cfo, ery, pen & wound ${ }^{*}$ & 1 \\
\hline 13 & 15 & IV & oxa, cfo, ery, pen & wound & G \\
\hline 14 & 16 & IV & oxa, cfo, ery, pen & nasal & C \\
\hline 15 & 17 & IV & oxa, cfo, ery, pen & wound & C \\
\hline
\end{tabular}

MRSA: methicillin-resistant Staphylococcus aureus; BHU: basic health units; oxa: oxacillin; cfo: cefoxitin; Ivx: levofloxacin; cli: clindamycin; gen: gentamicin; ery: erythromycin; pen: penicillin; *No nasal swab performed. ** We used letters of the alphabet instead of names of BHU.

of patients, $S$. aureus isolates from the nasal and wound swabs had the same resistance profile.

The MRSA isolates in this study carried type II $[n=6$ (33\%)] and type IV $[n=12(67 \%)]$ chromosomal cassettes and showed high levels of resistance to the quinolones, macrolides, and lincosamides. However, in the present study, $p v l$ was not detected in methicillin-resistant strains, only in methicillinsensitive strains, a finding consistent with the low prevalence of $p v l$ in MRSA isolates in Brazil, which are mainly type IV. The findings also demonstrate that sulfamethoxazole-trimethoprim remains a good treatment choice, as the MRSA isolates showed $100 \%$ susceptibility. Other community-based studies of patients in the same region have described the same characteristics in circulating clones ${ }^{13}$.

Age was independently associated with a reduced risk of $S$. aureus presence, with a $6 \%$ decrease in the risk of carrying $S$. aureus for every additional year of age. One possible explanation is that as age increases, patients are exposed to factors (not assessed in this study) that prevent $S$. aureus colonization; conversely, it may be attributed to increased ecological competition with other microorganisms.
The use of amoxicillin (usually with concomitant use of clavulanate) and ciprofloxacin is also protective against acquisition of $S$. aureus; fluoroquinolone exposure is related to an increased risk of methicillin-resistant, not methicillinsensitive, isolates ${ }^{14}$. Prior benzylpenicillin use was a risk factor for $S$. aureus carriage; benzylpenicillin is one of the most commonly used drugs in primary care, especially for the treatment of chronic wound infection. Consistent with our results, Staphylococcus spp. worldwide currently show high resistance $(\geq 80 \%)$ to penicillin G. After analyzing nearly 29,000 nasal swabs from healthy individuals from eight European countries, van Bijnen et al. ${ }^{15}$ described an association between frequent penicillin prescription and high odds of nasal carriage of resistant S. aureus. We also observed a high frequency of penicillin use during our analysis of patient records. Penicillin results in Streptococcus death and S. aureus permanence.

Some of the limitations of this study include the lack of identification of other putative pathogens; difficulty in differentiating infection from colonization in chronic wounds, an issue that remains controversial; failure to include certain 
Wounds

171 patients

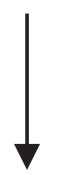

98 S. aureus

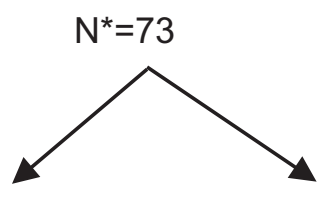

14 MRSA

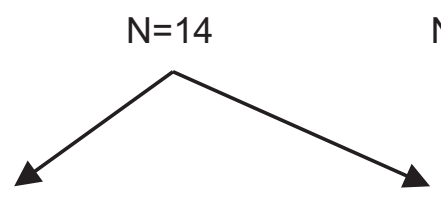

5 SCCmec II
9 SCCmec IV
Nasal

74 patients

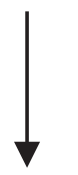

21 S. aureus
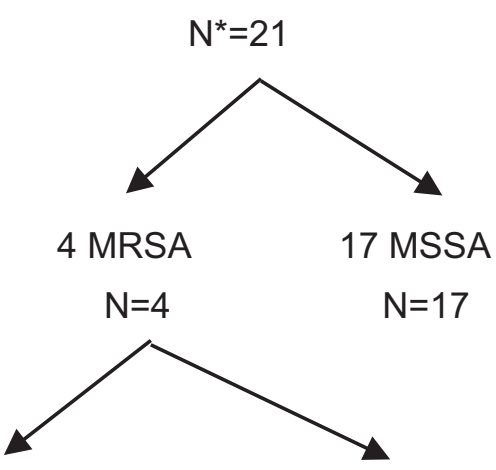

1 SCCmec II

3 ScCmec IV

FIGURE 1 - Flowchart showing the number of $S$. aureus isolates obtained from nasal and wound swabs. S. aureus: Staphylococcus aureus; MRSA: methicillin-resistant Staphylococcus aureus; MSSA: methicillin-sensitive Staphylococcus aureus; SCCmec: staphylococcal cassete chromosome $m e c$; ${ }^{*}$ Number of patients.

characteristics, such as lesion diameter, in the questionnaire; and not collecting nasal samples from all study participants.

However, ours is one of the first studies on the prevalence of $S$. aureus and MRSA in patients with chronic wounds managed at various BHU in Brazil for a period of almost three years. Our results point to a high prevalence of $S$. aureus and the presence of MRSA isolates that are resistant to several classes of antimicrobials, suggesting the possible dissemination of these pathogens within the primary care services in the city studied. These findings call attention to the circulation and potential reservoir of resistant strains in patients without the usual risk factors or exposures particular to the hospital setting. They also demonstrate the need for more research in to be conducted in these types of healthcare facilities to understand the prevalence and circulation dynamics of multidrug-resistant microorganisms, and the possible existence of healthcare-related infections.

\section{Ethical considerations}

The authors assert that all procedures related to this work complied with local ethical standards and the Declaration of Helsinki (1975). Patients signed informed consent forms prior to application of the questionnaire. This study was approved by the Ethics Committee of Botucatu Medical School-UNESP (CEP 3127-2009 and 3958-2011).

\section{Acknowledgments}

We express our deepest thanks to the patients attending the basic health units (BHUs) in Botucatu, Brazil.
Conflicts of interest

The authors declare that there is no conflict of interest.

\section{Financial support}

This study was supported by the São Paulo Research Foundation [Fundação de Amparo `s Pesquisa do Estado de São Paulo (FAPESP)]; grant numbers 2011/10146-7, 2012/00257-9, 2013/10975-9.

\section{REFERENCES}

1. Almeida GCM, Santos MM, Lima NGM, Cidral TA, Melo MCN, Lima KC. Prevalence and factors associated with wound colonization by Staphylococcus spp. and Staphylococcus aureus in hospitalized patients in inland northeastern Brazil: a cross-sectional study. BMC Infect Dis. 2014;14:328.

2. Levine NS, Lindberg RB, Mason AD, Pruitt BA. The quantitative swab culture and smear: a quick, simple method for determining the number of viable aerobic bacteria on open wound. J Trauma. 1976;16(2):89-94.

3. Pereira EPL, Cunha MLRS. Avaliação da colonização nasal por Staphylococcus spp. resistente à oxacilina em alunos de enfermagem. J Bras Patol Med Lab. 2009;45(5):361-9.

4. Winn WC, Allen SD, Janda WM, Koneman E, Procop G, Schreckenberger P, Woods G. Gram - Positive Cocci: Part I. In: Winn WC, Allen SD, Janda WM, Koneman E, Procop G, Schreckenberger P, Woods G, editors. Color Atlas and Textbook of Diagnostic Microbiology. 6th edition. Philadelphia: Lippincott Williams and Wilkins; 2006. p. 623-671. 
5. Clinical and Laboratory Standards Institute (CLSI). Performance Standards for Antimicrobial Susceptibility Testing; Twenty-Fifth Informational Supplement. CLSI document M100-S25. CLSI; 2015. $240 \mathrm{p}$.

6. Maor Y, Rahav G, Belausov N, Ben-David D, Smollan G, Keller N. Prevalence and characteristics of heteroresistant vancomycinintermediate Staphylococcus aureus bacteremia in a tertiary care center. J Clin Microbiol. 2007;45(5):1511-4.

7. McDonald RR, Antonishyn NA, Hansen T, Snook LA, Nagle E, Mulvey MR, et al. Development of a triplex real time PCR assay for detection of panton-valentine leukocidin toxin genes in clinical isolates of methicillin-resistant Staphylococcus aureus. J Clin Microbiol. 2005;43(12):6147-9.

8. Milheiriço C, Oliveira DC, Lencastre H. Update to the multiplex PCR strategy for assignment of mec element types in Staphylococcus aureus. Antimicrob Agents Chemother. 2007;51(9):3374-7.

9. Wiley Online Library. Wound infection in clinical practice. An international consensus. Special Issue Supplement. Int Wound J. 2008;5(s3):iii-11. doi:10.1111/j.1742-481X.2008.00488.x.

10. Forcade NA, Parchman ML, Jorgensen JH, Du CL, Nyren NR, Treviño LB, et al. Prevalence, severity, and treatment of communityacquired methicillin-resistant Staphylococcus aureus (CA-MRSA) skin and soft tissue infections in 10 medical clinics in Texas:
A South Texas Ambulatory Research Network (STARNet) Study. J Am Board Fam Med. 2011;24(5):543-50.

11. Martins MA, Tipple AFV, Reis C, Santiago SB, Bachion MM. Úlcera crônica de perna de pacientes em tratamento ambulatorial: análise microbiológica e de suscetibilidade antimicrobiana. Cienc Cuid Saude. 2010;9(3):464-70.

12. Martins MA, Santos SLV, Leão LSNO, Araújo NP, Bachion MM. Prevalence of resistance phenotypes in Staphylococcus aureus and coagulase-negative isolates of venous ulcers of primary healthcare patients. Rev Soc Bras Med Trop. 2012;45(6):717-22.

13. Bonesso MF, Marques SA, Camargo $\mathrm{CH}$, Fortaleza CMCB, Cunha MLRS. Community-associated methicillin-resistant Staphylococcus aureus in non-outbreak skin infections. Braz J Microbiol. 2014;45(4):1401-7.

14. Weber SG, Gold HS, Hooper DC, Karchmer AW, Carmeli Y. Fluoroquinolones and the risk for methicillin-resistant Staphylococcus aureus in hospitalized patients. Emerg Infect Dis. 2003;9(11):1415-22.

15. van Bijnen EME, Paget J, de Lange-de Klerk ESM, den Heijer CDJ, Verspoten A, Stobberingh EE, et al. Antibiotic exposure another risk factors for antimicrobial resistance in nasal commensal Staphylococcus aureus: an ecological study in 8 European countries. PLoS one. 2015;10(8):e0135094. 